\title{
The quality of antimicrobial prescribing in acute care hospitals: results derived from a national point prevalence survey, Germany, 2016
}

Seven Johannes Sam Aghdassi ${ }^{1,2}$, Frank Schwab ${ }^{1,2}$, Sonja Hansen ${ }^{1,2}$, Luis Alberto Peña Diaz ${ }^{1,2}$, Michael Behnke ${ }^{1,2}$, Petra

Gastmeier ${ }^{1,2}$, Tobias Siegfried Kramer ${ }^{1,2}$

1. Charité-Universitätsmedizin Berlin, corporate member of Freie Universität Berlin, Humboldt-Universität zu Berlin and Berlin

Institute of Health, Institute of Hygiene and Environmental Medicine, Berlin, Germany

2. National Reference Centre for Surveillance of Nosocomial Infections, Berlin, Germany

Correspondence: Seven Johannes Sam Aghdass (seven-johannes-sam.aghdassi@charite.de)

Citation style for this article:

Aghdassi Seven Johannes Sam, Schwab Frank, Hansen Sonja, Peña Diaz Luis Alberto, Behnke Michael, Gastmeier Petra, Kramer Tobias Siegfried. The quality of antimicrobial prescribing in acute care hospitals: results derived from a national point prevalence survey, Germany, 2016. Euro Surveill. $2019 ; 24(46)$ :pii=1900281. https://doi.org/10.2807/1560-7917.ES.2019.24.46.1900281

Background: Robust data on the quality of antimicrobial prescriptions in German acute care hospitals are scarce. To establish and implement antimicrobial stewardship (AMS) measures and to increase prudent antimicrobial use (AMU), the identification of appropriate process and quality indicators is pertinent. Aim: Our main objective was to identify parameters associated with adequate $A M U$ and inadequate $A M U$ by analysing point prevalence data. Our secondary goal was to describe the current state of AMS implementation in Germany. Methods: A national point prevalence survey for healthcare-associated infections and AMU was conducted in German hospitals in 2016. Data on structure and process parameters were also collected. Recorded antimicrobial prescriptions were divided into adequate, inadequate and undefinable AMU. A multivariable linear regression analysis was performed to examine the correlation of selected structure and process parameters with the adequacy of recorded antimicrobials. Results: Data from 218 acute care hospitals, 64,412 patients and 22,086 administered antimicrobials were included. Multivariable linear regression analysis revealed that documentation of a reason for $\mathrm{AMU}$ in the patient notes increased the likelihood of adequate $A M U$ and decreased the likelihood of inadequate $A M U$ significantly $(p<0.001)$, while tertiary care hospital type had the opposite effect ( $p<0.001)$. Conclusion: Through associating structural and process parameters with adequacy of $A M U$, we identified parameters that increased the odds of prudent AMU. Documentation was a key element for improving AMU. Revealed deficits regarding the implementation of AMS in German hospitals concerning dedicated staff for AMS activities and establishment of regular AMU training and AMU audits should be tackled.

\section{Introduction}

Healthcare systems worldwide have attempted to establish antimicrobial stewardship (AMS) programmes since the concept of AMS was introduced over 20 years ago [1]. AMS focuses on improving prescription practices of antimicrobials to improve outcomes in patients with infectious diseases through more effective treatment, and to reduce adverse effects. Such aims have been shown to be achievable for instance, through reduction and timely discontinuation of ineffective or prolonged antimicrobial treatments [2]. Recent data on the prevalence of antimicrobial use (AMU) in German hospitals revealed a shift in the most frequently administered antimicrobials to a higher proportion of broad-spectrum antimicrobials [3], which generally are associated with more adverse effects than antimicrobials with a narrower spectrum.

To promote prudent use of antimicrobials in German hospitals, various measures have been proposed [4-6]. In 2013, a national guideline on the prudent use of antimicrobials in hospitals was published [7]. Among other measures, the guideline recommended the implementation of interdisciplinary AMS teams with designated staff in every hospital. Many hospitals in Germany have introduced some features of AMS, but the level of implementation varies widely [8].

The term 'never events' is used to describe errors that should never occur in medical practice [9]. Although identifying never events for antimicrobials poses a challenge, attempts have been made in this respect $[10,11]$. Defining inadequate AMU (i.e. never events) from point prevalence data can be a means to achieve this goal. 
Box 1

Definitions of antimicrobial applications' adequacy ${ }^{\mathrm{a}}$

Antimicrobial applications defined as adequate:

- surgical prophylaxis for not more than 24 hours $[6,33]$,

- AMU for treatment of community-acquired pneumonia with a treatment duration of 7 days or shorter $[6,34]$, and

- AMU for treatment of pyelonephritis with de-escalation of the initial treatment or switch to oral treatment $[35,36]$.

Antimicrobial applications defined as inadequate:

- surgical prophylaxis for more than 24 hours $[6,33]$,

- AMU for treatment of community-acquired pneumonia with a treatment duration of more than 7 days $[6,34,37]$,

- AMU for treatment of pyelonephritis without de-escalation of the initial treatment or switch to oral treatment $[35,36]$,

- AMU for treatment of asymptomatic bacteriuria $[6,7,36]$, and

- AMU without clear (i.e. unknown) indication $[6,7]$.

All other antimicrobial applications were considered undefinable.

AMU: antimicrobial use.

a Based on available literature.

The main objectives of this study were to describe and analyse the current state of antimicrobial prescription quality in German acute care hospitals that participated in the national point prevalence survey (PPS) of healthcare-associated infections $(\mathrm{HAI})$ and $\mathrm{AMU}$ in 2016, and to associate the data with a set of structural and process parameters, which were recorded as part of the survey. Furthermore, as a secondary objective, we aimed to describe the current state of AMS implementation in German acute care hospitals.

\section{Methods}

A cross-sectional PPS was conducted in acute care hospitals in Germany between May and June 2016. All 1,462 hospitals participating in the German nosocomial infection surveillance system 'Krankenhaus-InfektionsSurveillance-System' (KISS) as of the first quarter of 2016, and other acute care hospitals in Germany were invited to participate on a voluntary basis.

\section{Data collection}

Data collectors were local hospital staff trained in methodology and HAl definitions at special one-day courses, to ensure methodological consistency. The data were collected according to the methodology and definitions provided by the European Centre for Disease Prevention and Control (ECDC). The light protocol version 5.1 of the ECDC PPS protocol was implemented [12].
All data gathered in the PPS and used in our analyses were from variables included in the ECDC PPS protocol, such as data on $\mathrm{HAl}$ and $\mathrm{AMU}$, patient-related data for patients with an active $\mathrm{HAl}$ and/or receiving at least one antimicrobial on the day of the survey, as well as structural and process parameters at the hospital and ward level. The original data collection sheets used and information on all data collected can be found in the ECDC PPS protocol [12]. Additional variables, which were not outlined in the protocol, were not collected. Specifics of data collection and management of German PPS data were described previously in more detail [13].

For AMU, the Anatomical Therapeutic Chemical Classification System of the World Health Organization [14] was used. Data on antimicrobials' route of application, indication for use, duration of application, documentation of a reason for AMU in the patient notes, modification of treatment and dosage, were recorded.

\section{Analyses of antimicrobial use}

While the primary endpoint of the PPS was to estimate the prevalence of patients with $\mathrm{HAl}$, the prevalence of patients with AMU was a secondary endpoint. Collection of data concerning AMU and structural characteristics of participating hospitals allowed further AMU-related analyses. In our study, we investigated the quality of antimicrobial prescriptions and possible associations with hospitals' structural and process parameters, as specified by the ECDC protocol [12]. In order to do this, the adequacy of every antimicrobial application was determined and allocated to one of three categories: adequate, inadequate or undefinable. This allocation was based on available literature, as specified in Box 1.

We then calculated the rate of adequate AMU as the number of adequate antimicrobial applications per 100 definable (i.e. adequate plus inadequate) applications, thereby excluding undefinable AMU from the denominator. In an analogous manner, we calculated the rate of inadequate $A M U$ as the number of inadequate antimicrobial applications per 100 definable applications.

Further analyses were performed to identify structural and process parameters associated with an increase in the rate of adequate or inadequate AMU. To determine either outcome, we performed a univariable and a multivariable linear regression analysis. The multivariable analysis was conducted by variable selection stepwise forward, with a p value 0.05 for a parameter to be included in the model and a $p$ value $<0.06$ for a parameter to remain in the model.

The structural and process parameters included in the univariable analysis are given in Box 2. Parameters were also included in the multivariable linear regression analysis, except those where datasets were incomplete. The parameters hospital type and hospital 


\section{Box 2}

Parameters included in the univariable and multivariable linear regression analyses ${ }^{\mathrm{a}}$

The following structural and process parameters were included in the analyses:

- hospital size (i.e. number of beds: $<300, \geq 300$ ),

- bed occupancy (\%) on the day of the PPS and as a yearly mean (i.e. number of patient days per year divided by number of available hospital bed-days per year),

- hospital type ${ }^{b}$ (primary care, secondary care, tertiary care, specialised hospital),

- hospital ownership ${ }^{b}$ (public, private (not for profit), private (for profit), other/unknown),

- number of blood cultures per 100 patient days,

- number of stool samples for CDI per 100 patient days,

- participation in surveillance networks for CDI,

- participation in surveillance networks for antimicrobial consumption and resistance,

- implementation of key bundles and multimodal strategies for selected AMS aspects,

- percentage of beds with systematic review routines for prescribed antimicrobials within 72 hours,

- equipment with designated AMS personnel,

- prevalence of patients with AMU, and

- percentage of administered antimicrobials with a reason documented in the patient notes.

AMS: antimicrobial stewardship; AMU: antimicrobial use; CDI: Clostridioides difficile infection; ECDC: European Centre for Disease Prevention and Control; PPS: point prevalence survey.

a The following parameters where datasets were incomplete were not used in the multivariable linear regression analysis: number of blood cultures per 100 patient days, number of stool samples for CDI per 100 patient days, percentage of beds with systematic review routines for prescribed antimicrobials within 72 hours and equipment with designated AMS personnel.

b The parameters hospital type and hospital ownership were collected in alignment with the ECDC PPS protocol [12] and analysed as dummycoded parameters in the univariable and multivariable analyses.

ownership were analysed as dummy-coded parameters in the univariable and multivariable analyses.

\section{Ethical approval}

The German Protection against Infection Act (Infektionsschutzgesetz) requires hospitals in Germany to collect surveillance data on $\mathrm{HAI}$ and AMU. All data used in our analyses and presented in this study are surveillance-based data. Ethical approval or informed consent were therefore not required.

\section{Results}

A total of 218 hospitals participated in the PPS (Table 1). Data from 64,412 patients and 22,086 prescribed antimicrobials were included in the analyses. The overall prevalence of patients receiving at least one antimicrobial was $26 \%$. Only 61 hospitals (28\%) stated that there was designated AMS staff employed at the hospital site, with nine hospitals reporting one or more AMS full-time equivalent staff members (FTE). A mean number of 0.1 AMS FTE per 250 hospital beds were recorded (median 0.0). A descriptive analysis of structural and process parameters of AMU can be found in Table 2 .

Results of the multivariable linear regression analysis for an increase in the rate of adequate AMU and inadequate AMU are illustrated in Table 4; corresponding univariable analyses can be found in the supplementary material (Table S1, Table S2). Documentation of a reason for AMU in the patient notes was a parameter associated with both a significant increase in the rate of adequate $A M U$ and a significant decrease in the rate of inadequate AMU. Tertiary care hospital type showed the opposite association.

\section{Discussion}

Key components of AMS are the identification and reduction of improper antimicrobial prescriptions $[2,15,16]$. In our study, we were able to distinguish adequate from inadequate antimicrobial prescriptions using point prevalence data and to associate adequate and inadequate AMU with structural and process parameters.

Through our multivariable linear regression analysis, documentation of a reason for $A M U$ in the patient notes was identified as a factor to both increase the likelihood of adequate AMU and decrease the risk of inadequate $A M U$. We consider this to be our most conclusive and relevant finding. To ensure a continuously high standard in the practice of prescribing antimicrobials, good documentation that enables all healthcare 
TABLE 1

Structural characteristics of hospitals participating in the point prevalence survey, Germany, $2016(\mathrm{n}=218)$

\begin{tabular}{|c|c|c|c|}
\hline Variable & Group/parameter & $\begin{array}{c}\text { Number or } \\
\text { median }\end{array}$ & $\%$ or IQR \\
\hline \multirow{4}{*}{ Hospital type } & Primary care & 118 & 54.1 \\
\hline & Secondary care & 41 & 18.8 \\
\hline & Tertiary care & 36 & 16.5 \\
\hline & Specialised hospital & 23 & 10.6 \\
\hline \multirow{4}{*}{ Hospital ownership } & Public & 103 & 47.2 \\
\hline & Private, not for profit & 63 & 28.9 \\
\hline & Private, for profit & 31 & 14.2 \\
\hline & Other/unknown & 21 & 9.6 \\
\hline \multirow{5}{*}{ Region in Germany } & East $^{\mathrm{a}}$ & 43 & $19 \cdot 7$ \\
\hline & South-east ${ }^{b}$ & 27 & 12.4 \\
\hline & South-west ${ }^{c}$ & 56 & $25 \cdot 7$ \\
\hline & North $^{d}$ & 29 & $13 \cdot 3$ \\
\hline & West $^{\mathrm{e}}$ & 63 & 28.9 \\
\hline \multirow{2}{*}{ Hospital size } & $<300$ beds & 105 & 48.2 \\
\hline & $\geq 300$ beds & 113 & 51.8 \\
\hline Patient days & Patient days per year & 81,586 & $46,448.0-144,014.0$ \\
\hline Bed occupancy per 100 beds & On the day of survey & 75 & $68.5-82.0$ \\
\hline
\end{tabular}

IQR: interquartile range.

a Berlin, Brandenburg, Saxony, Saxony-Anhalt and Thuringia.

b Bavaria and Hesse.

' Baden-Württemberg, Saarland and Rhineland-Palatinate.

${ }^{\mathrm{d}}$ Bremen, Hamburg, Lower Saxony, Mecklenburg-West Pomerania and Schleswig-Holstein.

e North Rhine-Westphalia.

workers to understand why an antimicrobial is administered is of high importance [17]. Documenting a reason in a patient's notes indicates that the prescriber put thought and reasoning into the prescription and helps others to better understand and evaluate the prescription, as well as effectively modify it when necessary $[18,19]$.

In recent years, the concept of bundle strategies has been integrated into medical practice including AMS activities $[20,21]$. For $A M U$, one of the most effective elements of such a bundle strategy is the use of checklists [22,23]. Our data corroborated this by demonstrating that the existence of a checklist for AMU at the hospital level was associated with $>11 \%$ reduction in the rate of inadequate AMU.

Another interesting finding of our multivariable regression analysis was that the variable tertiary care hospital type significantly reduced the likelihood of adequate antimicrobial applications and significantly increased the likelihood of inadequate applications. This may in part be attributable to differences in patient populations. Patients treated in tertiary care hospitals generally suffer from more severe diseases and, as a consequence, receive more complex treatments [24]. Furthermore, tertiary care hospitals usually employ a higher number of medical doctors whose rotations are more frequent, which could lead to a higher degree of discontinuity in diagnosis and treatment. Another parameter significantly associated with lowering the rate of adequate AMU was high bed occupancy. This finding suggests that a higher workload due to a higher density of patients may lead to less adequate antimicrobial prescribing behaviour, which is possibly less thoughtful and more rushed. A contributing factor may be the link between patient overcrowding (i.e. high bed occupancy) and a more frequent occurrence of HAI [25], which as a result leads to more complicated treatment regimens.

As a secondary objective, this study sought to describe of the state of AMS implementation in German hospitals. Our survey revealed that, as of 2016 , less than a third of participating hospitals had designated staff for AMS and only nine hospitals reported dedicating one or more FTE for AMS, which confirms the discrepancy between recommendations [26] and their current implementation. When compared with other European countries [27,28], we consider the current AMS staffing situation and state of implementation of AMS measures in Germany critical and insufficient, at least in the participating hospitals. This is relevant because the training of AMS experts and installation of interdisciplinary AMS teams have shown to improve antimicrobial prescription practices [29]. 
Structural and process parameters of antimicrobial use and antimicrobial stewardship at hospitals participating in the point prevalence survey, Germany, $2016(\mathrm{n}=218)$

\begin{tabular}{|c|c|c|c|}
\hline Variable & Group/parameter & $\begin{array}{l}\text { Number or } \\
\text { median }\end{array}$ & $\%$ or IQR \\
\hline \multirow[b]{2}{*}{ Microbiological diagnostics } & Number of blood cultures per 100 patient days & 2.1 & $1.3-3.1$ \\
\hline & $\begin{array}{c}\text { Number of stool samples for Clostridioides difficile infection } \\
\text { per } 100 \text { patient days }\end{array}$ & 0.7 & $0.5-1.1$ \\
\hline \multirow{3}{*}{ Surveillance (regional or national network) } & C. difficile infection & 119 & 54.6 \\
\hline & Antimicrobial consumption & 83 & 38.1 \\
\hline & AMR & 56 & 25.7 \\
\hline \multirow{7}{*}{$\begin{array}{l}\text { Components of multimodal strategies (at the } \\
\text { hospital level) }\end{array}$} & Guideline for AMU & 157 & 72.0 \\
\hline & Training for AMU & 37 & 17.0 \\
\hline & Bundle for AMU & 136 & 62.4 \\
\hline & Checklist for AMU & 25 & 11.5 \\
\hline & Audit for AMU & 74 & 33.9 \\
\hline & Surveillance of AMU & 145 & 66.5 \\
\hline & Feedback of data on AMU to frontline HCW & 150 & 64.2 \\
\hline $\begin{array}{l}\text { Post-prescription review of antimicrobials } \\
\text { within } 72 \text { hours }\end{array}$ & Percentage of hospital beds & 0 & $0.0-16.8$ \\
\hline \multirow{3}{*}{$\begin{array}{l}\text { Designated staff for antimicrobial } \\
\text { stewardship }\end{array}$} & Hospital with designated staff for AMS & 61 & 28.0 \\
\hline & Full-time equivalents per hospital & o & $0.0-0.1$ \\
\hline & Full-time equivalents per 250 beds & o & $0.0-0.1$ \\
\hline
\end{tabular}

AMR: antimicrobial resistance; AMS: antimicrobial stewardship; AMU: antimicrobial use; HCW: healthcare workers; IQR: interquartile range.

The German Protection against Infection Act requires hospitals to evaluate and interpret their antimicrobial consumption at least annually and, if necessary, to implement measures to improve consumption accordingly [5]. Over $60 \%$ of participating hospitals in our survey reported adherence to this requirement, with also over $60 \%$ providing regular feedback on AMU to frontline healthcare workers. This suggests a steady increase in adherence compared with earlier studies $[8,30]$. Surprisingly, only $38 \%$ of hospitals reported participation in structured surveillance networks (regional or national) for antimicrobial consumption, although these are easily accessible and participation is free of costs in Germany [31]. We found this to be an area for potential improvement.

At the facility level, training and education for AMU, as well as local guidelines on empirical antimicrobial treatment, are important factors for improving AMU quality $[4,32]$. However, data on how healthcare facilities, and in particular acute care hospitals, adhere to these recommendations are scarce. Our data revealed considerable opportunities for improvement, as only $17 \%$ of hospitals regularly undertook AMU training and only around a third of participating hospitals had established AMU audits. Our data suggest that the implementation of AMS measures in Germany, as defined by national recommendations [7], is still far from being achieved.

\section{Limitations}

Our study had several limitations. The data used for our analyses were chosen with the objective of identifying structure and process parameters to evaluate antimicrobial prescriptions. The PPS that was conducted in 2016 was not primarily intended for such analyses. Instead, its primary focus was to estimate the prevalence of patients with HAl. However, other variables collected in the PPS allow for careful secondary analyses, as they provide valuable information on structural characteristics of participating hospitals. Another important limitation was that data collection in participating hospitals was performed by a heterogeneous group of professionals with substantial differences regarding experience in conducting surveillance and, more importantly for our analysis, differences in knowledge about antimicrobial prescription. Therefore, inconsistencies in data collection and recording that might confound the data cannot be excluded. However, by ensuring that data collectors were trained systematically before the survey, the data should have gained robustness and consistency with regard to the definitions and methodology applied. In order to categorise the recorded $\mathrm{AMU}$ into adequate and inadequate applications, we had to choose parameters and criteria from the limited data available. Furthermore, for the majority of antimicrobial applications recorded, such a categorisation was not possible; these applications were not attributed to either group, but remained undefinable. 
TABLE 3

Antimicrobial use in hospitals participating in the point prevalence survey, Germany, $2016(\mathrm{n}=218)$

\begin{tabular}{|c|c|c|c|}
\hline Variable & Group/parameter & $\begin{array}{c}\text { Number or } \\
\text { median }\end{array}$ & $\%$ or IQR \\
\hline \multirow{2}{*}{ Number of recorded antimicrobials } & All hospitals & 22,086 & 100 \\
\hline & Per hospital & $64 \cdot 5$ & $38.0-125.0$ \\
\hline \multirow{2}{*}{ Number of observed patients } & All hospitals & 64,412 & 100 \\
\hline & Per hospital & 220.5 & $122.0-377.0$ \\
\hline Prevalence of patients with AMU & All hospitals & 26.2 & $19.5-30.5$ \\
\hline \multirow{3}{*}{ Documentation of a reason for AMU in the patient notes } & All hospitals & 15,165 & 68.7 \\
\hline & Per hospital & 41.5 & $20.0-85.0$ \\
\hline & Per 100 recorded antimicrobials & 77.5 & $54 \cdot 3-90.9$ \\
\hline \multirow{4}{*}{ Adequate applications of antimicrobials } & All hospitals & 3,349 & 15.2 \\
\hline & Per hospital & 12 & $4.0-21.0$ \\
\hline & Per 100 antimicrobials & 16 & $9.0-24.6$ \\
\hline & Per 100 definable antimicrobials & $55 \cdot 3$ & $35.8-71.4$ \\
\hline \multirow{4}{*}{ Inadequate applications of antimicrobials } & All hospitals & 3,872 & $17 \cdot 5$ \\
\hline & Per hospital & 9 & $4.0-21.0$ \\
\hline & Per 100 antimicrobials & 16.7 & $10 \cdot 5-23 \cdot 5$ \\
\hline & Per 100 definable antimicrobials & 50 & $35.4-66.7$ \\
\hline \multirow{3}{*}{ Undefinable applications of antimicrobials } & All hospitals & 14,865 & 67.3 \\
\hline & Per hospital & 41 & $24.0-83.0$ \\
\hline & Per 100 antimicrobials & 67.1 & $59 \cdot 3-74.8$ \\
\hline
\end{tabular}

AMU: antimicrobial use; IQR: interquartile range.

TABLE 4

Multivariable linear regression analysis by rates of adequate and inadequate antimicrobial applications per 100 definable antimicrobial applications of hospitals participating in the point prevalence survey, Germany, $2016(\mathrm{n}=218)$

\begin{tabular}{|c|c|c|c|}
\hline Outcome & Parameter & $\mathrm{p}$ value & $\begin{array}{l}\text { Regression coefficient } \\
(95 \% \mathrm{Cl})\end{array}$ \\
\hline \multirow{3}{*}{$\begin{array}{l}\text { Rate of adequate antimicrobial applications per } \\
100 \text { definable applications }\end{array}$} & $\begin{array}{l}\text { Documentation of a reason for AMU in the patient notes } \\
\text { (per increase of } 1 \% \text { ) }\end{array}$ & $<0.001$ & $0.22(0.10$ to 0.34$)$ \\
\hline & Bed occupancy as a yearly mean (per increase of $1 \%$ ) & 0.046 & $-0.32(-0.63$ to -0.01$)$ \\
\hline & Tertiary care hospital type & 0.001 & $-14.51(-22.78$ to -6.24$)$ \\
\hline \multirow{3}{*}{$\begin{array}{l}\text { Rate of inadequate antimicrobial applications } \\
\text { per } 100 \text { definable applications }\end{array}$} & Checklist for antimicrobial use (at the hospital level) & 0.018 & $-11.54(-21.09$ to -2.00$)$ \\
\hline & $\begin{array}{l}\text { Documentation of a reason for AMU in the patient notes } \\
\text { (per increase of } 1 \% \text { ) }\end{array}$ & $<0.001$ & $-0.23(-0.34$ to -0.11$)$ \\
\hline & Tertiary care hospital type & $<0.001$ & $14.80(6.57$ to 23.03$)$ \\
\hline
\end{tabular}

AMU: antimicrobial use; $\mathrm{Cl}$ : confidence interval.

We consider the number of included hospitals $(n=218)$, although not drawn from a representative sample, to be a strength of our study, which allows for careful extrapolations to the national level.

\section{Conclusions}

This study demonstrated the important role of documentation as a factor for improving the quality of AMU. Contrarily, the variables tertiary care hospital type and high bed occupancy were associated with a decrease in adequate $A M U$, which indicates that a higher workload may be a barrier for the prudent use of antimicrobials.
The results also illustrated deficits in the implementation of AMS in German acute care hospitals, in particular with regard to AMS staffing, training for AMU and participation in networks for antimicrobial consumption, which should be tackled. Future studies should focus on novel approaches to utilise point prevalence data to evaluate antimicrobial prescription practices and barriers to successful AMS implementation.

Acknowledgements 
The authors wish to thank all of the hospitals that participated in the national point prevalence survey for their efforts and dedication. Moreover, we wish to extend thanks to the European Centre for Disease Prevention and Control for initiating and supporting the survey on the European level and the German Ministry of Health for funding the survey. The German Ministry of Health funded the national point prevalence survey on healthcare-associated infections and antimicrobial use in 2016 (grant number ZMVI1-2515FSB101).

\section{Conflict of interest}

None declared.

\section{Authors' contributions}

Seven Johannes Sam Aghdassi and Tobias Siegfried Kramer drafted the manuscript and determined the content and objectives of the analyses. Frank Schwab conducted the statistical analysis. Luis Alberto Peña Diaz and Michael Behnke managed the data storage of the point prevalence survey and extracted the analysed data sets from the data storage. Petra Gastmeier, Sonja Hansen and Seven Johannes Sam Aghdassi led the organisation of the point prevalence survey. All authors have read and approved the final version of the manuscript.

\section{References}

1. Charani E, Holmes A. Antibiotic Stewardship-Twenty Years in the Making. Antibiotics (Basel). 2019;8(1):E7. https://doi. org/10.3390/antibiotics8010007 PMID: 30678365

2. Dyar OJ, Huttner B, Schouten J, Pulcini CESGAP (ESCMID Study Group for Antimicrobial stewardshiP). What is antimicrobial stewardship? Clin Microbiol Infect. 2017;23(11):793-8. https:// doi.org/10.1016/j.cmi.2017.08.026 PMID: 28882725

3. Aghdassi SJS, Gastmeier P, Piening BC, Behnke M, Peña Diaz LA, Gropmann A, et al. Antimicrobial usage in German acute care hospitals: results of the third national point prevalence survey and comparison with previous national point prevalence surveys. J Antimicrob Chemother. 2018;73(4):1077-83. https:// doi.org/10.1093/jac/dkx494 PMID: 29309607

4. Lübbert C, Schumacher U, Stareprawo S, Claus J, Heeß-Erle $\mathrm{G}$, Fiebig $\mathrm{C}$, et al. [Can the antibiotic prescription practice in a hospital be influenced by in-house guidelines? An interventional study at the University Hospital Halle (Saale), Germany]. Dtsch Med Wochenschr. 2014;139(50):2578-84. PMID: 25271804

5. Schweickert B, Kern WV, de With K, Meyer E, Berner R, Kresken $M$, et al. [Surveillance of antibiotic consumption : clarification of the "definition of data on the nature and extent of antibiotic consumption in hospitals according to $\S 23$ paragraph 4 sentence 2 of the IfSG"]. Bundesgesundheitsblatt Gesundheitsforschung Gesundheitsschutz. 2013;56(7):903-12. https://doi.org/10.1007/s00103-013-1764-8 PMID: 23807401

6. Thern J, de With K, Strauss R, Steib-Bauert M, Weber N, Kern WV. Selection of hospital antimicrobial prescribing quality indicators: a consensus among German antibiotic stewardship (ABS) networkers. Infection. 2014;42(2):351-62. https://doi. org/10.1007/S15010-013-0559-Z PMID: 24326986

7. de With K, Allerberger F, Amann S, Apfalter P, Brodt HR, Eckmanns T, et al. Strategies to enhance rational use of antibiotics in hospital: a guideline by the German Society for Infectious Diseases. Infection. 2016;44(3):395-439. https:// doi.org/10.1007/S15010-016-0885-Z PMID: 27066980

8. Maechler F, Schwab F, Geffers C, Meyer E, Leistner R, Gastmeier P. Antibiotic stewardship in Germany: a crosssectional questionnaire survey of 355 intensive care units. Infection. 2014;42(1):119-25. https://doi.org/10.1007/S15010013-0531-y PMID: 24135909

9. Kumar J, Raina R. ‘Never Events in Surgery': Mere Error or an Avoidable Disaster. Indian J Surg. 2017;79(3):238-44. https:// doi.org/10.1007/s12262-017-1620-4 PMID: 28659678

10. Glowacki RC, Schwartz DN, Itokazu GS, Wisniewski MF, Kieszkowski P, Weinstein RA. Antibiotic combinations with redundant antimicrobial spectra: clinical epidemiology and pilot intervention of computer-assisted surveillance. Clin Infect Dis. 2003;37(1):59-64. https://doi.org/10.1086/376623 PMID: 12830409

11. Liu J, Kaye KS, Mercuro NJ, Davis SL, Patel TS, Petty LA, et al. It is time to define antimicrobial never events. Infect Control Hosp Epidemiol. 2019;40(2):206-7. https://doi.org/10.1017/ ice.2018.313 PMID: 30516126

12. European Centre for Disease Prevention and Control (ECDC). Point prevalence survey of healthcare-associated infections and antimicrobial use in European acute care hospitals. Protocol version 5.3. Stockholm: ECDC; 2016. Available from: https://ecdc.europa.eu/sites/portal/files/media/en/ publications/Publications/PPS-HAI-antimicrobial-use-EUacute-care-hospitals-V5-3.pdf

13. Behnke M, Aghdassi SJ, Hansen S, Diaz LAP, Gastmeier $P$, Piening B. The Prevalence of Nosocomial Infection and Antibiotic Use in German Hospitals. Dtsch Arztebl Int. 2017;114(50):851-7. https://doi.org/10.3238/arztebl.2017.0851 PMID: 29271343

14. WHO Collaborating Centre for Drug Statistics Methodology (WHOCC). Guidelines for ATC classification and DDD assignment 2013. Oslo: WHOCC; 2012. Available from: https:// www.whocc.no/filearchive/publications/1_2013guidelines.pdf

15. Charani E, Castro-Sanchéz E, Bradley S, Nathwani D, Holmes $\mathrm{AH}$, Davey P. Implementation of antibiotic stewardship in different settings - results of an international survey. Antimicrob Resist Infect Control. 2019;8(1):34. https://doi. org/10.1186/s13756-019-0493-7 PMID: 30805181

16. Nathwani D, Varghese D, Stephens J, Ansari W, Martin S, Charbonneau C. Value of hospital antimicrobial stewardship programs [ASPs]: a systematic review. Antimicrob Resist Infect Control. 2019;8(1):35. https://doi.org/10.1186/s13756-0190471-0 PMID: 30805182

17. Woodford EM, Wilson KA, Marriott JF. Documentation of antibiotic prescribing controls in UK NHS hospitals. J Antimicrob Chemother. 2004;53(4):650-2. https://doi. org/10.1093/jac/dkh152 PMID: 15014063

18. Katsios CM, Burry L, Nelson S, Jivraj T, Lapinsky SE, Wax RS, et al. An antimicrobial stewardship program improves antimicrobial treatment by culture site and the quality of antimicrobial prescribing in critically ill patients. Crit Care. 2012;16(6):R216. https://doi.org/10.1186/cc11854 PMID: 23127353

19. Pulcini C, Dellamonica J, Bernardin G, Molinari N, Sotto A. Impact of an intervention designed to improve the documentation of the reassessment of antibiotic therapies in an intensive care unit. Med Mal Infect. 2011;41(10):54652. https://doi.org/10.1016/j.medmal.2011.07.003 PMID: 21855239

20. Moody J, Cosgrove SE, Olmsted R, Septimus E, Aureden K, Oriola S, et al. Antimicrobial stewardship: a collaborative partnership between infection preventionists and healthcare epidemiologists. Infect Control Hosp Epidemiol. 2012;33(4):328-30. https://doi.org/10.1086/665037 PMID: 22418626

21. Schuts EC, Hulscher MEJL, Mouton JW, Verduin CM, Stuart JWTC, Overdiek HWPM, et al. Current evidence on hospital antimicrobial stewardship objectives: a systematic review and meta-analysis. Lancet Infect Dis. 2016;16(7):847-56. https:// doi.org/10.1016/S1473-3099(16)00065-7 PMID: 26947617

22. van Daalen FV, Prins JM, Opmeer BC, Boermeester MA, Visser $C E$, van Hest RM, et al. Effect of an antibiotic checklist on length of hospital stay and appropriate antibiotic use in adult patients treated with intravenous antibiotics: a stepped wedge cluster randomized trial. Clin Microbiol Infect. 2017;23(7):485. e1-8. https://doi.org/10.1016/j.cmi.2017.01.019 PMID: 28159671

23. van Daalen FV, Prins JM, Opmeer BC, Boermeester MA, Visser CE, van Hest RM, et al. A cluster randomized trial for the implementation of an antibiotic checklist based on validated quality indicators: the AB-checklist. BMC Infect Dis. 2015;15(1):134. https://doi.org/10.1186/s12879-015-0867-2 PMID: 25888180

24. Kuster SP, Ruef C, Bollinger AK, Ledergerber B, Hintermann $A$, Deplazes $C$, et al. Correlation between case mix index and antibiotic use in hospitals. J Antimicrob Chemother. 2008;62(4):837-42. https://doi.org/10.1093/jac/dkn275 PMID: 18617509

25. Kaier K, Mutters NT, Frank U. Bed occupancy rates and hospital-acquired infections--should beds be kept empty? Clin Microbiol Infect. 2012;18(10):941-5. https://doi.org/10.1111/ j.1469-0691.2012.03956.x PMID: 22757765

26. May M, Vetterlein MW, Wagenlehner FM, Brookman-May SD, Gilfrich C, Fritsche HM, et al. [What is the perception of the 10 -point plan of the German Federal Ministry of Health against multidrug-resistant pathogens and measures of antibiotic 
stewardship?] Urologe A. 2017;56(10):1302-10. https://doi. org/10.1007/s00120-017-0425-1 PMID: 28593351

27. Kallen MC, Binda F, Ten Oever J, Tebano G, Pulcini C, Murri R, et al. Comparison of antimicrobial stewardship programmes in acute-care hospitals in four European countries: A crosssectional survey. Int J Antimicrob Agents. 2019;54(3):338-45. https://doi.org/10.1016/j.ijantimicag.2019.06.005 PMID: 31200022

28. Perozziello A, Lescure FX, Truel A, Routelous C, Vaillant L, Yazdanpanah Y, et al. Prescribers' experience and opinions on antimicrobial stewardship programmes in hospitals: a French nationwide survey. J Antimicrob Chemother. 2019;74(8):2451-8. https://doi.org/10.1093/jac/dkz179 PMID: 31167027

29. Christoph A, Ehm C, de With K. [Impact of an $|A| B|S|$-training initiative on $|\mathrm{A}| \mathrm{B}|\mathrm{S}|$-structural quality of participating hospitals]. Z Evid Fortbild Qual Gesundhwes. 2015;109(7):521-7. https://doi.org/10.1016/j.zefq.2015.09.021 PMID: 26593768

30. Chaberny IF, Wriggers A, Behnke M, Gastmeier P. Antibiotics: MRSA prevention measures in German hospitals: results of a survey among hospitals, performed as part of the MRSA-KISS module. Dtsch Arztebl Int. 2010;107(37):631-7. PMID: 20959890

31. Schweickert B, Feig M, Schneider M, Willrich N, Behnke $M$, Peña Diaz LA, et al. Antibiotic consumption in Germany: first data of a newly implemented web-based tool for local and national surveillance. J Antimicrob Chemother. 2018;73(12):3505-15. https://doi.org/10.1093/jac/dky345 PMID: 30239728

32. Davey P, Marwick CA, Scott CL, Charani E, McNeil K, Brown $E$, et al. Interventions to improve antibiotic prescribing practices for hospital inpatients. Cochrane Database Syst Rev. 2017;2:CDo03543. https://doi.org/10.1002/14651858. CDo03543.pub4 PMID: 28178770

33. Allegranzi B, Bischoff P, de Jonge S, Kubilay NZ, Zayed B, Gomes SM, et al. New WHO recommendations on preoperative measures for surgical site infection prevention: an evidencebased global perspective. Lancet Infect Dis. 2016;16(12):e27687. https://doi.org/10.1016/S1473-3099(16)30398-X PMID: 27816413

34. Ewig S, Höffken G, Kern WV, Rohde G, Flick H, Krause R, et al. [Management of Adult Community-acquired Pneumonia and Prevention - Update 2016]. Pneumologie. 2016;70(3):151-200. PMID: 26926396

35. van den Bosch CM, Geerlings SE, Natsch S, Prins JM, Hulscher ME. Quality indicators to measure appropriate antibiotic use in hospitalized adults. Clin Infect Dis. 2015;60(2):281-91. https:// doi.org/10.1093/cid/ciu747 PMID: 25266285

36. Kranz J, Schmidt S, Lebert C, Schneidewind L, Mandraka F, Kunze M, et al. The 2017 Update of the German Clinical Guideline on Epidemiology, Diagnostics, Therapy, Prevention, and Management of Uncomplicated Urinary Tract Infections in Adult Patients. Part II: Therapy and Prevention. Urol Int. 2018;100(3):271-8. https://doi.org/10.1159/000487645 PMID: 29539622

37. Onakpoya IJ, Walker AS, Tan PS, Spencer EA, Gbinigie OA, Cook J, et al. Overview of systematic reviews assessing the evidence for shorter versus longer duration antibiotic treatment for bacterial infections in secondary care. PLoS One. 2018;13(3):e0194858. https://doi.org/10.1371/journal. pone.0194858 PMID: 29590188

\section{License, supplementary material and copyright}

This is an open-access article distributed under the terms of the Creative Commons Attribution (CC BY 4.0) Licence. You may share and adapt the material, but must give appropriate credit to the source, provide a link to the licence and indicate if changes were made.

Any supplementary material referenced in the article can be found in the online version.

This article is copyright of the authors or their affiliated institutions, 2019. 